\title{
Automated High-Throughput Partition Coefficient Determination with Image Analysis For Rapid Reaction Workup Process Development and Modeling.
}

Sophie Duffield†, Luigi Da Vià*§, Amelia Celeste Bellman§, Fabio Chiti†

$\S C M C$ Analytical, $†$ Chemical Development

GlaxoSmithKline, Pharmaceutical Research and Development, Medicinal Science \& Technology

Stevenage, Gunnels Wood Road, Stevenage Herts, SG1 2NY

\section{Supporting Information}

\section{Automated Protocol}

The Unchained Labs Freeslate CM3 (Unchained Labs, Pleasanton, CA, USA) is a versatile automated platform capable of performing several unit operations (e.g., solid dispense, liquid addition, and $\mathrm{pH}$ measurement) due to the flexible configuration of the deck elements and of the robotic arms. Typically, solid, and liquid dispensing capabilities are default modules as the robot is capable of dispensing solids in the range of 0.01-100 $\mathrm{g}$ depending on the requirement of the workflow. The solids are weighed using different tools based on the dispense range and are weighed on the on-deck 4 figures balance. The balance module also hosts a camera which can take pictures of vials at selected timepoints (Figure S1).

In terms of liquid transfer, the platform offers several options with the use of the inline piercing needles connected to $5 \times 1 \mathrm{~mL}$ and $1 \times 2.5 \mathrm{~mL}$ syringes and with the use of positive displacement tips (PDT). By using a combination of these features, it is possible to dispense liquid in the $25-10000 \mu \mathrm{L}$ range.

The platform can also host several bays to heat/cool plates and the typical operating range is between 5 $80^{\circ} \mathrm{C}$ but this range can be extended based on the chiller configuration and the fluid used. Stirring of vials and plates can be performed with the magnetic tumble stirrer or by using the vortexing module. The latter, hosts an Inheco Thermoshake AC (Inheco, $\mathrm{GmbH}$, Germany) which is capable of stirring multiwell plates in the range 300-3000 rpm and between $4-70^{\circ} \mathrm{C}$.

The CM3 is fitted with 2 multifunctional arms which can be configured to support a variety of different workflows. In this case, the platform is fitted with a multifunctional gripper able to move plates and individual vials across the deck, a 4 solid state $\mathrm{pH}$ probes modules, a universal adapter (to pick up solid and dispensing tools), and with the 6 spreadable tip module (Figure S2). The variable pitch configuration of the 6-tip module enables the platform to perform up to 6 liquid dispenses at once across a variety of different plates formats. 


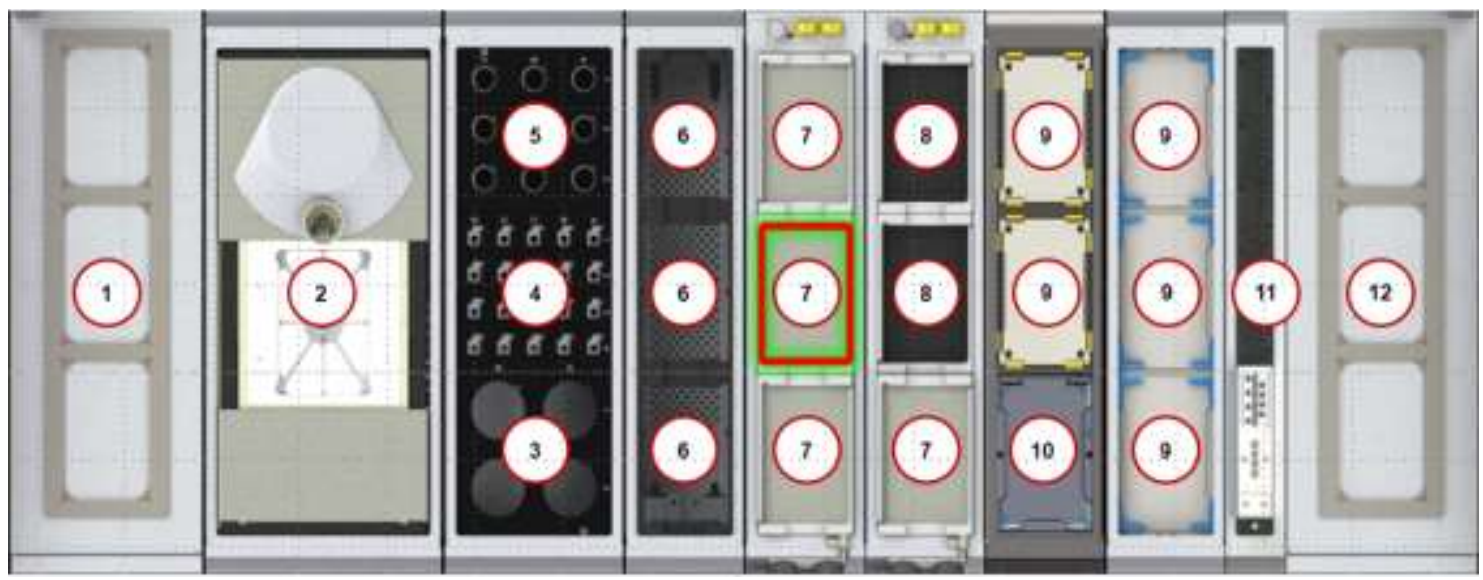

Figure S1. Detailed overview of the deck of the Freeslate CM3

The CM3 deck configuration shown in Figure S1 highlights all the positions on deck:

1. Waste container for used plastic tips

2. Analytical balance

3. Classic hopper storage positions; Training tool and SV tool storage positions

4. Classic hoppers storage positions

5. Positive displacement tip (PDT) tools storage positions

6. $3 x$ PDT racks storage positions

7. Temperature controlled magnetic stirred bay (within a range of $-5-80^{\circ} \mathrm{C}$ )

8. Magnetic stirred position (not temperature controlled)

9. 5x Room temperature vortexers (independently controlled)

10. $1 \mathrm{x}$ Heated vortexer (independently controlled)

11. $\mathrm{pH} \&$ needles washing station +4

12. $3 \mathrm{x}$ plates storage positions off deck

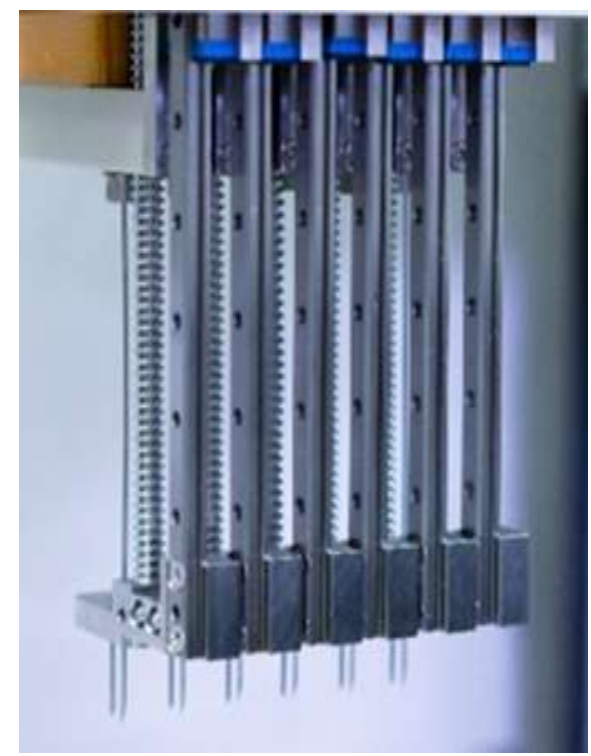

Figure S2. Spreadable 6-tip arm 


\subsection{Solid Dosing}

All APIs and reagents were weighed into the $8 \mathrm{~mL}$ vials using the Freeslate CM3, Mettler Toledo QX96 or Mettler Toledo QB30 systems depending on the powder properties the right powder dosing system was chosen. Depending on the powder properties the most suitable powder dosing system was chosen. Typically, the Mettler Toledo Pinocchio head (QH012-LNMP) and the Unchained Labs SV hoppers (SG151057-10) were used.

\subsection{Image analysis}

The Freeslate was programmed to transfer the vials from the well plate to the on-deck balance with an integrated camera, using the vial/plate gripper arm. Images were taken at multiple time points, just before sampling the phases. The images taken were run through the MATLAB script - further details of this can be found in the section 3. Image analysis development below.

\subsection{Density measurement samples}

The density of each phase is calculated by sampling a known volume, $500 \mu \mathrm{L}$, of each organic and aqueous phase into pre-tared vials and weighed again after sampling. Sampling was performed on the Freeslate using the spreadable 6-Tip (Figure S2) and vials were weighed on the on-deck balance.

The density values are to calculate the phase masses which are combined with the NMR, HPLC and KF data to obtain a full mass balance.

\subsection{UHPLC analysis}

The concentration of APIs and known impurities is determined by HPLC against standard calibration curves. Typically, standard calibration samples are prepared by dispensing a few $\mathrm{mg}$ of material in clear glass vials. The solids are then dissolved using the appropriate solvent the to achieve a final concentration of $1000 \mu \mathrm{g} / \mathrm{mL}$. This sample is then further diluted to generate the other analytical standards at 250, 62.5, and $15.62 \mu \mathrm{g} / \mathrm{mL}$ respectively.

Solubility of the samples was measured using an Agilent 1290 system with a photodiode array detector (DAD). A Waters XBridge $\mathrm{C} 18(50 \times 4.6 \mathrm{~mm}, 3.5 \mu \mathrm{m})$ column kept at $30^{\circ} \mathrm{C}$ was used with aqueous (mobile phase A) $10 \mathrm{mM}$ aqueous ammonium bicarbonate adjusted to $\mathrm{pH} 10$ with aqueous ammonia and organic (mobile phase B) acetonitrile in gradient with a flowrate of $3.3 \mathrm{~mL} / \mathrm{min}$.

Different dilutions were prepared where appropriate (usually 10x and 100x dilutions) by sampling $10 \mu \mathrm{L}$ from the $500 \mu \mathrm{L}$ density measurement samples and dissolving in $990 \mu \mathrm{L}$ 3:1 acetonitrile:water containing $0.065 \mathrm{mg} / \mathrm{mL}$ of biphenyl as an internal standard.

\subsection{KF analysis}

Empty $2 \mathrm{~mL}$ screw cap HPLC vials were tared on the Freeslate balance and re-weighed again after adding $500 \mu \mathrm{L}$ methanol and again after adding the analytical sample (organic or aqueous phase). For organic samples, $100 \mu \mathrm{L}$ from the $500 \mu \mathrm{L}$ density measurement samples was dissolved in $400 \mu \mathrm{L}$ organic and for the aqueous phase $100 \mu \mathrm{L}$ sample was dissolved in $900 \mu \mathrm{L}$ methanol. 


\section{6. ${ }^{1} \mathrm{H}-\mathrm{NMR}$ analysis}

The samples were prepared from the $500 \mu \mathrm{L}$ density measurement samples, taking $100 \mu \mathrm{L}$ and dissolving in $600 \mu \mathrm{L}$ of $\mathrm{d}_{6}$-DMSO with TMS. For analysing reaction components, the solvent was removed using a rotary evaporator. For analysing residual solvents, this step was skipped.

The samples were analysed by ${ }^{1} \mathrm{H}$ or $1 \mathrm{HQuant}$ methods on a Bruker AVIIIHD Nanobay $400 \mathrm{MHz}$ openaccess NMR with a $5 \mathrm{~mm}$ BBO probe with the following parameters.

- Field: $400.1 \mathrm{MHz}$

- Acquisition time $4.06 \mathrm{~s}$

- Relaxation delay: $10.0 \mathrm{~s}$

- Pulse program: zg30

- Temperature: $300.0 \mathrm{~K}$

\section{Verification of Automated Protocol (ternary phase diagram)}

KF analysis was carried out on neat samples using a Mettler-Toledo V10S volumetric Karl Fischer with reagent Hydranal ${ }^{\mathrm{TM}}$ Composite $5 \mathrm{~K}$ and medium Hydranal ${ }^{\mathrm{TM}}$ Medium $\mathrm{K}$.

${ }^{1} \mathrm{H}$ NMR analysis of samples was carried out in $\mathrm{d}_{6}$-DMSO using a Bruker AVIIIHD Nanobay $400 \mathrm{MHz}$ NMR with a $5 \mathrm{~mm}$ BBO probe with the following parameters.

- Field: $400.1 \mathrm{MHz}$

- Acquisition time $2.03 \mathrm{~s}$

- Relaxation delay: $10.0 \mathrm{~s}$

- Pulse program: zg30

- Temperature: $300.0 \mathrm{~K}$

Controlled lab reactor (CLR) data was collected by adding $100 \mathrm{~mL}$ lab grade water and $100 \mathrm{ml}$ reagent grade 2-methyltetrahydrofuran (2MeTHF) to a $500 \mathrm{~mL}$ Reactor Ready jacketed CLR. The mixture was agitated at $300 \mathrm{rpm}$ for 30 minutes with a jacket temperature of $25^{\circ} \mathrm{C}$, then left to settle with no agitation. Both phases were sampled for KF and NMR measurements. The mixture composition was then adjusted by adding $10 \mathrm{~mL}$ of 2-propanol and the procedure repeated. Aliquots of 2-propanol were added until a single phase was obtained. 
Table S1. Organic and aqueous phase compositions generate from the conventional CLR methodology, and two runs using the Freeslate CMC3 LLE Protocol.

\begin{tabular}{|c|c|c|c|c|c|c|c|c|}
\hline \multicolumn{9}{|c|}{ Organic Phase Results (wt\%) } \\
\hline \multicolumn{3}{|c|}{ CLR RESULTS } & \multicolumn{3}{|c|}{ CM3 RESULTS $1^{\text {st }}$ RUN } & \multicolumn{3}{|c|}{ CM3 RESULTS $2^{\text {nd }}$ RUN } \\
\hline Water & 2MeTHF & 2-Propanol & Water & 2MеTHF & 2-Propanol & Water & 2МеТНF & 2-Propanol \\
\hline 4.4 & 95.6 & 0 & 4.9 & 95.1 & 0 & 4.6 & 95.4 & 0 \\
\hline 8.3 & 86.3 & 5.4 & 8.4 & 86.2 & 5.4 & 9 & 85.3 & 5.7 \\
\hline 13.2 & 76.4 & 10.5 & 12.9 & 76.6 & 10.5 & 13.6 & 75.1 & 11.2 \\
\hline 19.9 & 65.3 & 14.8 & 18.6 & 66.4 & 15 & 19 & 65.4 & 15.6 \\
\hline 28.3 & 53.9 & 17.8 & 24.1 & 58.9 & 17 & 26.4 & 54.1 & 19.5 \\
\hline \multicolumn{3}{|c|}{ Single Phase } & 37.1 & 42.5 & 20.4 & 39.4 & 40.4 & 20.2 \\
\hline \multicolumn{9}{|c|}{ Aqueous Phase Results (wt\%) } \\
\hline \multicolumn{3}{|c|}{ CLR RESULTS } & \multicolumn{3}{|c|}{ CM3 RESULTS $1^{\text {st }}$ RUN } & \multicolumn{3}{|c|}{ CM3 RESULTS $2^{\text {nd }}$ RUN } \\
\hline Water & 2MeTHF & 2-Propanol & Water & 2MeTHF & 2-Propanol & Water & 2MeTHF & 2-Propanol \\
\hline 88.8 & 11.2 & 0 & 83.9 & 16.1 & 0 & 84.8 & 15.2 & 0 \\
\hline 82.4 & 13.8 & 3.8 & 79.8 & 15.8 & 4.4 & 81.4 & 13.2 & 5.4 \\
\hline 78.5 & 14.9 & 6.6 & 74.3 & 17.4 & 8.3 & 82.9 & 9.7 & 7.4 \\
\hline 77.8 & 14 & 8.3 & 73.1 & 16.9 & 10 & 72.3 & 14.7 & 13 \\
\hline 72.8 & 16.5 & 10.7 & 70.4 & 17.1 & 12.5 & 70 & 16 & 13.9 \\
\hline 44.1 & 37.4 & 18.5 & 56.4 & 27.6 & 16 & 59.6 & 23.8 & 16.6 \\
\hline
\end{tabular}

\section{Image Analysis Development}

The filters that are applied as part of the image analysis code operate as follows.

- If more than $62 \%$ of pixels in a row are below the gradient threshold the entire row is considered to not contain part of the meniscus (no "black band" applied)

- For rows identified as being part of the meniscus, a check is made of whether additional rows have been identified within 10 pixels; if so, they are all considered to be part of the same meniscus (multiple small black bands replaced with one larger band).

- The bottom portion of the vial which is affected by reflections from the base is excluded from the meniscus identification.

- The pixel height reported depends on whether the quadratic curve fitted to the meniscus has a minimum or a maximum. If it has a minimum, the pixel at this minimum is the value reported (i.e. "bottom of the meniscus"). However, due to the perspective of the vial, the liquid/air interface often shows a maximum rather than a minimum. In this case, reporting the pixel position as the point at which the fitted curve intersects the left border of the image was found to give a better fit.

Materials used in generating the coloured water and heptane solutions for testing the robustness of the image analysis code were as follows.

Materials:

The chemicals used in the image analysis study were received from Sigma-Aldrich and used without any further purification. The colorants were dissolved in laboratory grade water and in heptane. 
A list of the components used and the colours obtained is reported below:

Yellow aqueous: 4-nitrophenol

Orange aqueous: Eosin Y

Green aqueous: Copper (II) sulfate, $\mathrm{NaCl}$

Blue aqueous: Copper (II) sulfate

Yellow organic: beta-carotene

Pink organic: $\quad$ Sudan Red 7B

Blue organic: Azulene

Raw data on the measured volumes from the process images are include in Table $\mathbf{S 2}$ below.

Table S2. Pixel and volume values calculated by the MATLAB image analysis code

\begin{tabular}{|c|c|c|c|c|c|c|c|c|}
\hline $\begin{array}{c}\text { Array } \\
\text { Position }\end{array}$ & $\begin{array}{c}\text { Organic } \\
\text { Volume } \\
(\mathrm{mL})\end{array}$ & $\begin{array}{l}\text { Aqueous } \\
\text { Volume } \\
\text { (mL) }\end{array}$ & $\begin{array}{c}\text { Organic } \\
\text { Pixels }\end{array}$ & $\begin{array}{c}\text { Aqueous } \\
\text { Pixels }\end{array}$ & $\begin{array}{c}\text { Calculated } \\
\text { Organic } \\
\text { Volume }(\mathrm{mL})\end{array}$ & $\begin{array}{c}\text { Calculated } \\
\text { Aqueous } \\
\text { Volume }(\mathrm{mL})\end{array}$ & $\begin{array}{c}\text { Organic } \\
\text { Error } \\
(\mathrm{mL})\end{array}$ & $\begin{array}{l}\text { Aqueous } \\
\text { Error }(\mathrm{mL})\end{array}$ \\
\hline A1 & 1 & 4 & 44.96 & 180.36 & 0.995 & 3.992 & -0.005 & -0.008 \\
\hline $\mathrm{A} 2$ & 1 & 4 & 42.33 & 183.60 & 0.937 & 4.064 & -0.063 & 0.064 \\
\hline A3 & 1 & 4 & 48.85 & 178.04 & 1.081 & 3.941 & 0.081 & -0.059 \\
\hline A4 & 1 & 4 & 41.51 & 184.47 & 0.919 & 4.083 & -0.081 & 0.083 \\
\hline A5 & 1 & 4 & \multicolumn{6}{|c|}{ N/A - no liquid/liquid interface detected } \\
\hline A6 & 1 & 4 & 50.40 & 175.73 & 1.116 & 3.890 & 0.116 & -0.110 \\
\hline B1 & 2 & 3 & 90.96 & 134.40 & 2.013 & 2.975 & 0.013 & -0.025 \\
\hline B2 & 2 & 3 & 91.45 & 135.92 & 2.024 & 3.008 & 0.024 & 0.008 \\
\hline B3 & 2 & 3 & 91.94 & 136.36 & 2.035 & 3.018 & 0.035 & 0.018 \\
\hline B4 & 2 & 3 & 92.78 & 132.54 & 2.054 & 2.934 & 0.054 & -0.066 \\
\hline B5 & 2 & 3 & \multicolumn{6}{|c|}{ N/A - no liquid/liquid interface detected } \\
\hline B6 & 2 & 3 & 95.72 & 133.22 & 2.119 & 2.949 & 0.119 & -0.051 \\
\hline $\mathrm{C} 1$ & 3 & 2 & 138.84 & 87.10 & 3.073 & 1.928 & 0.073 & -0.072 \\
\hline $\mathrm{C} 2$ & 3 & 2 & 138.15 & 88.65 & 3.058 & 1.962 & 0.058 & -0.038 \\
\hline $\mathrm{C} 3$ & 3 & 2 & 136.26 & 91.31 & 3.016 & 2.021 & 0.016 & 0.021 \\
\hline $\mathrm{C} 4$ & 3 & 2 & 139.22 & 88.09 & 3.081 & 1.950 & 0.081 & -0.050 \\
\hline $\mathrm{C} 5$ & 3 & 2 & 136.05 & 90.08 & 3.011 & 1.994 & 0.011 & -0.006 \\
\hline $\mathrm{C} 6$ & 3 & 2 & 139.74 & 86.80 & 3.093 & 1.921 & 0.093 & -0.079 \\
\hline D1 & 4 & 1 & \multicolumn{6}{|c|}{ N/A - no liquid/liquid interface detected } \\
\hline D2 & 4 & 1 & 185.57 & 42.81 & 4.107 & 0.948 & 0.107 & -0.052 \\
\hline D3 & 4 & 1 & 183.35 & 43.31 & 4.058 & 0.959 & 0.058 & -0.041 \\
\hline D4 & 4 & 1 & \multicolumn{6}{|c|}{ N/A - no liquid/liquid interface detected } \\
\hline D5 & 4 & 1 & \multicolumn{6}{|c|}{ N/A - no liquid/liquid interface detected } \\
\hline D6 & 4 & 1 & \multicolumn{6}{|c|}{ N/A - incorrect interface position identified } \\
\hline
\end{tabular}




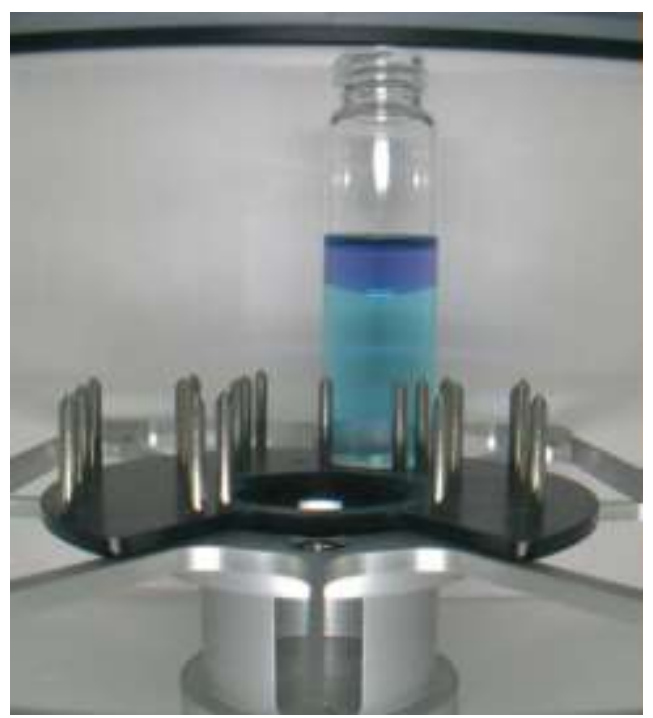

Figure S3. Example of raw image of a vial from the Freeslate deck camera before being cropped for the MatLab code.

\section{Case Study}

HPLC analysis of samples was carried out by adding $10 \mu \mathrm{L}$ of each sample to $1 \mathrm{ml}$ of diluent. The diluent was 3:1 acetonitrile:water containing $0.065 \mathrm{mg} / \mathrm{mL}$ of biphenyl as an internal standard. The column was Waters XBridge $\mathrm{C} 18(50 \times 4.6 \mathrm{~mm}, 3.5 \mu \mathrm{m})$ at $30^{\circ} \mathrm{C}$ with a flow rate of $3.0 \mathrm{~mL} / \mathrm{min}$. Mobile phase A was $10 \mathrm{mM}$ aqueous ammonium bicarbonate adjusted to $\mathrm{pH} 10$ with aqueous ammonia; mobile phase B was acetonitrile. The gradient was as follows:

Table S3. HPLC method gradient details.

\begin{tabular}{|c|c|c|}
\hline Time (mins) & $\mathbf{\% A}$ & $\mathbf{\% B}$ \\
\hline 0.00 & 99 & 1 \\
\hline 0.10 & 99 & 1 \\
\hline 4.00 & 3 & 97 \\
\hline 5.00 & 3 & 97 \\
\hline 5.01 & 99 & 1 \\
\hline 7.00 & 99 & 1 \\
\hline
\end{tabular}

Samples were run at a $1.0 \mu \mathrm{L}$ injection volume and wavelength of $220 \mathrm{~nm}$.

${ }^{1} \mathrm{H}$ NMR analysis of samples was carried out in $\mathrm{d}_{4}$-methnaol using a Bruker AVIIIHD Nanobay $400 \mathrm{MHz}$ NMR with a $5 \mathrm{~mm}$ BBO probe with the following parameters.

- Field: $400.1 \mathrm{MHz}$

- Acquisition time $4.06 \mathrm{~s}$

- Relaxation delay: $10.0 \mathrm{~s}$

- Pulse program: zg30

- Temperature: $300.0 \mathrm{~K}$

KF analysis was carried out using a Mettler-Toledo V10S volumetric Karl Fischer as detailed above for case study 1. Organic layer samples were analysed neat; aqueous layer samples were diluted with dry methanol by a factor of 10 prior to analysis. 


\section{Case Study Mass Balance}

The combination of the above analytical techniques gave the following component quantifications for both phases:

- Total phase volume by image analysis

- Total phase mass by image analysis and density measurement

- Product in $\mathrm{mg} / \mathrm{ml}$ by HPLC, converted to $\mathrm{mg}$ using the total phase volume.

- Impurity in $\mathrm{mg} / \mathrm{ml}$ by HPLC, converted to $\mathrm{mg}$ using the total phase volume.

- Water in $\mathrm{mg} / \mathrm{g}$ by $\mathrm{KF}$, converted to $\mathrm{mg}$ using total phase mass

- Byproduct 1, 2, 3 and 4 in $\mathrm{mg} / \mathrm{g}$ by NMR (only relative to the other components measured by NMR)

- Toluene and Ethanol in mg/g by NMR (only relative to the other components measured by NMR)

The masses of product, impurity and water were then subtracted from the total phase mass to give the total mass of the components measured by NMR. The NMR calculated $\mathrm{mg} / \mathrm{g}$ values were then applied to this adjusted total mass, to give the masses on Byproducts 1-4, toluene and ethanol. Hence the measurements allow full quantification of every component; the resulting masses in each phase are given in Table S4 below. It should be noted that GC analysis as a replacement for NMR would provide further simplification, as it would generate values in $\mathrm{mg} / \mathrm{ml}$ which could by treated in the same way as HPLC measurements.

A similar approach was followed for the input component masses, with the proportion of the components in the reaction mixture quantified by HPLC and NMR and the masses calculated from the quantity of reaction mixture added to each vial. As water was also added directly to the vial prior to mixing to generate the biphase, the mass of this component was already known. The resulting input masses are shown in Table S5 below.

Table S4. Measured Component Masses in the Organic and Aqueous Phases

\begin{tabular}{|c|c|c|c|c|c|c|c|c|c|}
\hline \multirow{2}{*}{ Sample } & \multicolumn{9}{|c|}{ Measured Mass of Component (g) } \\
\cline { 2 - 11 } & Ethanol & Toluene & Water & Product & Impurity & Byproduct 1 & Byproduct 2 & Byproduct 3 & Byproduct 4 \\
\hline Plate 1, A1, Organic & 0.012 & 1.707 & 0.004 & 0.165 & 0.0043 & 0.030 & 0.045 & 0.010 & 0.060 \\
\hline Plate 1, A2, Organic & 0.024 & 2.110 & 0.015 & 0.225 & 0.0062 & 0.089 & 0.081 & 0.034 & 0.106 \\
\hline Plate 1, A3, Organic & 0.034 & 2.375 & 0.030 & 0.243 & 0.0068 & 0.188 & 0.113 & 0.071 & 0.131 \\
\hline Plate 1, B1, Organic & 0.062 & 1.377 & 0.009 & 0.112 & 0.0032 & 0.021 & 0.026 & 0.007 & 0.037 \\
\hline Plate 1, B2, Organic & 0.167 & 1.636 & 0.026 & 0.141 & 0.0043 & 0.063 & 0.051 & 0.024 & 0.071 \\
\hline Plate 1, B3, Organic & 0.312 & 1.686 & 0.064 & 0.161 & 0.0051 & 0.141 & 0.068 & 0.054 & 0.108 \\
\hline Plate 1, C1, Organic & 0.017 & 2.385 & 0.007 & 0.164 & 0.0059 & 0.011 & 0.012 & 0.002 & 0.019 \\
\hline Plate 1, C2, Organic & 0.015 & 2.543 & 0.006 & 0.183 & 0.0066 & 0.011 & 0.014 & 0.003 & 0.023 \\
\hline Plate 1, C3, Organic & 0.011 & 2.049 & 0.007 & 0.140 & 0.0050 & 0.008 & 0.008 & 0.002 & 0.014 \\
\hline Plate 1, C4, Organic & 0.007 & 1.768 & 0.008 & 0.095 & 0.0034 & 0.007 & 0.006 & 0.002 & 0.011 \\
\hline Plate 1, A1, Aqueous & 0.031 & 0.318 & 2.074 & 0.024 & 0.0012 & 0.164 & 0.071 & 0.059 & 0.087 \\
\hline Plate 1, A2, Aqueous & 0.045 & 0.207 & 1.269 & 0.019 & 0.0010 & 0.268 & 0.085 & 0.104 & 0.148 \\
\hline Plate 1, A3, Aqueous & 0.047 & 0.094 & 0.775 & 0.013 & 0.0007 & 0.301 & 0.071 & 0.119 & 0.206 \\
\hline Plate 1, B1, Aqueous & 0.265 & 0.433 & 2.002 & 0.038 & 0.0024 & 0.149 & 0.074 & 0.055 & 0.104 \\
\hline
\end{tabular}




\begin{tabular}{|c|c|c|c|c|c|c|c|c|c|}
\hline Plate 1, B2, Aqueous & 0.440 & 0.246 & 1.186 & 0.041 & 0.0021 & 0.252 & 0.106 & 0.093 & 0.179 \\
\hline Plate 1, B3, Aqueous & 0.532 & 0.205 & 0.596 & 0.046 & 0.0020 & 0.291 & 0.102 & 0.108 & 0.222 \\
\hline Plate 1, C1, Aqueous & 0.002 & 0.005 & 1.961 & 0.008 & 0.0006 & 0.001 & 0.002 & 0.000 & 0.001 \\
\hline Plate 1, C2, Aqueous & 0.018 & 0.032 & 1.645 & 0.017 & 0.0008 & 0.018 & 0.021 & 0.005 & 0.005 \\
\hline Plate 1, C3, Aqueous & 0.004 & 0.004 & 2.398 & 0.006 & 0.0000 & 0.004 & 0.004 & 0.001 & 0.001 \\
\hline Plate $1, \mathrm{C} 4$, Aqueous & 0.005 & 0.004 & 2.773 & 0.005 & 0.0000 & 0.006 & 0.005 & 0.001 & 0.002 \\
\hline Plate 2, A1, Organic & 0.019 & 1.619 & 0.012 & 0.137 & 0.0037 & 0.029 & 0.037 & 0.010 & 0.051 \\
\hline Plate 2, A2, Organic & 0.219 & 1.920 & 0.055 & 0.160 & 0.0046 & 0.136 & 0.070 & 0.052 & 0.110 \\
\hline Plate 2, A3, Organic & 0.054 & 1.481 & 0.006 & 0.122 & 0.0032 & 0.028 & 0.030 & 0.009 & 0.043 \\
\hline Plate 2, A4, Organic & 0.050 & 1.350 & 0.006 & 0.108 & 0.0027 & 0.019 & 0.021 & 0.006 & 0.030 \\
\hline Plate 2, B1, Organic & 0.112 & 1.911 & 0.020 & 0.159 & 0.0045 & 0.073 & 0.061 & 0.027 & 0.086 \\
\hline Plate 2, B2, Organic & 0.115 & 1.931 & 0.020 & 0.155 & 0.0045 & 0.075 & 0.064 & 0.028 & 0.088 \\
\hline Plate 2, B3, Organic & 0.170 & 1.663 & 0.031 & 0.124 & 0.0035 & 0.064 & 0.050 & 0.023 & 0.072 \\
\hline Plate 2, B4, Organic & 0.051 & 1.148 & 0.006 & 0.088 & 0.0022 & 0.016 & 0.017 & 0.005 & 0.024 \\
\hline Plate 2, $\mathrm{C} 1$, Organic & 0.011 & 2.127 & 0.005 & 0.134 & 0.0048 & 0.009 & 0.008 & 0.001 & 0.015 \\
\hline Plate 2, C2, Organic & 0.011 & 2.155 & 0.007 & 0.139 & 0.0049 & 0.009 & 0.009 & 0.001 & 0.015 \\
\hline Plate 2, C3, Organic & 0.021 & 1.895 & 0.005 & 0.114 & 0.0040 & 0.007 & 0.008 & 0.001 & 0.013 \\
\hline Plate 2, C4, Organic & 0.024 & 1.590 & 0.006 & 0.096 & 0.0032 & 0.007 & 0.007 & 0.001 & 0.011 \\
\hline Plate 2, A1, Aqueous & 0.088 & 0.048 & 2.116 & 0.026 & 0.0014 & 0.263 & 0.124 & 0.101 & 0.164 \\
\hline Plate 2, A2, Aqueous & 0.353 & 0.117 & 0.682 & 0.032 & 0.0014 & 0.328 & 0.111 & 0.123 & 0.250 \\
\hline Plate 2, A3, Aqueous & 0.293 & 0.046 & 2.021 & 0.030 & 0.0016 & 0.248 & 0.117 & 0.093 & 0.161 \\
\hline Plate 2, A4, Aqueous & 0.360 & 0.040 & 2.171 & 0.030 & 0.0016 & 0.230 & 0.113 & 0.084 & 0.149 \\
\hline Plate 2, B1, Aqueous & 0.302 & 0.058 & 1.190 & 0.030 & 0.0015 & 0.276 & 0.115 & 0.106 & 0.227 \\
\hline Plate 2, B2, Aqueous & 0.303 & 0.056 & 1.130 & 0.029 & 0.0014 & 0.290 & 0.120 & 0.110 & 0.240 \\
\hline Plate 2, B3, Aqueous & 0.522 & 0.079 & 1.099 & 0.040 & 0.0018 & 0.286 & 0.123 & 0.106 & 0.236 \\
\hline Plate 2, B4, Aqueous & 0.468 & 0.041 & 2.341 & 0.030 & 0.0016 & 0.192 & 0.100 & 0.070 & 0.148 \\
\hline Plate 2, C1, Aqueous & 0.004 & 0.002 & 2.308 & 0.006 & 0.0000 & 0.004 & 0.005 & 0.001 & 0.001 \\
\hline Plate 2, C2, Aqueous & 0.004 & 0.002 & 2.272 & 0.006 & 0.0000 & 0.004 & 0.005 & 0.001 & 0.001 \\
\hline Plate 2, C3, Aqueous & 0.052 & 0.005 & 2.455 & 0.009 & 0.0006 & 0.012 & 0.012 & 0.003 & 0.003 \\
\hline Plate 2, C4, Aqueous & 0.185 & 0.015 & 2.600 & 0.010 & 0.0007 & 0.027 & 0.025 & 0.006 & 0.008 \\
\hline
\end{tabular}

Table S5. Input Component Masses to Vials

\begin{tabular}{|c|c|c|c|c|c|c|c|c|c|}
\hline Sample & \multicolumn{9}{|c|}{ Input Mass of Component (g) } \\
\cline { 2 - 11 } & Ethanol & Toluene & Water & Product & Impurity & Byproduct 1 & Byproduct 2 & Byproduct 3 & Byproduct 4 \\
\hline Plate 1, A1 & 0.059 & 2.206 & 1.670 & 0.192 & 0.0056 & 0.318 & 0.136 & 0.039 & 0.241 \\
\hline Plate 1, A2 & 0.071 & 2.647 & 1.000 & 0.230 & 0.0067 & 0.381 & 0.163 & 0.047 & 0.290 \\
\hline Plate 1, A3 & 0.079 & 2.938 & 0.560 & 0.256 & 0.0075 & 0.423 & 0.181 & 0.052 & 0.322 \\
\hline Plate 1, B1 & 0.608 & 1.697 & 1.670 & 0.152 & 0.0052 & 0.265 & 0.111 & 0.032 & 0.237 \\
\hline Plate 1, B2 & 0.729 & 2.037 & 1.000 & 0.182 & 0.0062 & 0.318 & 0.133 & 0.038 & 0.284 \\
\hline Plate 1, B3 & 0.810 & 2.263 & 0.560 & 0.202 & 0.0069 & 0.353 & 0.148 & 0.042 & 0.316 \\
\hline Plate 1, C1 & 0.047 & 2.457 & 1.810 & 0.173 & 0.0063 & 0.030 & 0.033 & 0.003 & 0.045 \\
\hline
\end{tabular}




\begin{tabular}{|l|l|l|l|l|l|l|l|l|l|} 
Plate 1, C2 & 0.052 & 2.704 & 1.490 & 0.191 & 0.0069 & 0.033 & 0.036 & 0.004 & 0.049 \\
\hline Plate 1, C3 & 0.040 & 2.080 & 2.300 & 0.147 & 0.0053 & 0.025 & 0.028 & 0.003 & 0.038 \\
\hline Plate 1, C4 & 0.027 & 1.852 & 2.660 & 0.100 & 0.0036 & 0.017 & 0.019 & 0.002 & 0.026 \\
\hline Plate 2, A1 & 0.124 & 1.941 & 1.667 & 0.189 & 0.0059 & 0.300 & 0.117 & 0.034 & 0.470 \\
\hline Plate 2, A2 & 0.144 & 2.251 & 0.652 & 0.219 & 0.0069 & 0.348 & 0.136 & 0.040 & 0.545 \\
\hline Plate 2, A3 & 0.110 & 1.726 & 1.667 & 0.168 & 0.0053 & 0.267 & 0.104 & 0.031 & 0.418 \\
\hline Plate 2, A4 & 0.100 & 1.566 & 1.811 & 0.152 & 0.0048 & 0.242 & 0.094 & 0.028 & 0.380 \\
\hline Plate 2, B1 & 0.391 & 2.143 & 1.000 & 0.215 & 0.0068 & 0.310 & 0.127 & 0.040 & 0.527 \\
\hline Plate 2, B2 & 0.391 & 2.143 & 1.000 & 0.215 & 0.0068 & 0.310 & 0.127 & 0.040 & 0.527 \\
\hline Plate 2, B3 & 0.698 & 1.905 & 1.000 & 0.191 & 0.0061 & 0.276 & 0.113 & 0.035 & 0.468 \\
\hline Plate 2, B4 & 0.631 & 1.299 & 2.076 & 0.130 & 0.0041 & 0.188 & 0.077 & 0.024 & 0.319 \\
\hline Plate 2, C1 & 0.036 & 2.214 & 2.143 & 0.155 & 0.0056 & 0.026 & 0.026 & 0.002 & 0.037 \\
\hline Plate 2, C2 & 0.036 & 2.214 & 2.143 & 0.155 & 0.0056 & 0.026 & 0.026 & 0.002 & 0.037 \\
\hline Plate 2, C3 & 0.283 & 1.968 & 2.143 & 0.138 & 0.0050 & 0.023 & 0.023 & 0.002 & 0.033 \\
\hline Plate 2, C4 & 0.423 & 1.705 & 2.300 & 0.119 & 0.0043 & 0.020 & 0.020 & 0.002 & 0.029 \\
\hline
\end{tabular}

\section{Modeling Work}

When regressing the NRT1 model parameters for the background solvent system, only the first parameter pairs were regressed due to the limited data available with no temperature variation but were found to give an excellent fit.

Table S6. Regressed NRTL model parameters for the toluene / water / ethanol ternary solvent system at $25^{\circ} \mathrm{C}$. C parameter values were not regressed, but were fixed at the value shown in the table.

\begin{tabular}{|l|l|c|c|l|}
\hline Component 1 & Component 2 & $\mathbf{A}_{\mathbf{i j}}$ & $\mathbf{A}_{\mathbf{j i}}$ & $\mathbf{C}$ \\
\hline Toluene & Water & 4.139 & 8.589 & 0.2 \\
\hline Toluene & Ethanol & 0.168 & 1.041 & 0.3 \\
\hline Water & Ethanol & 2.198 & -1.607 & 0.3 \\
\hline
\end{tabular}




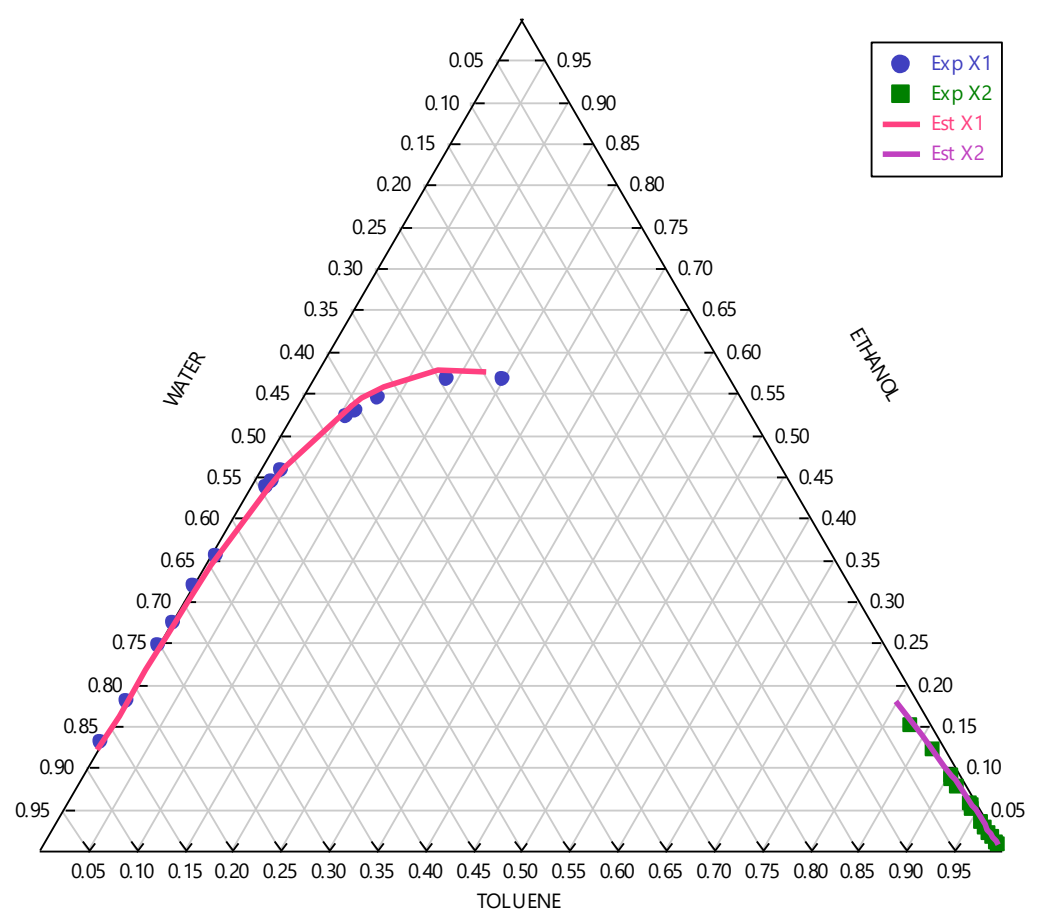

Figure S4. Ternary phase diagram showing the literature data (Exp) and NRTL model fit (Est) for mass fractions of the components in liquid phases X1 and X2.

When regressing the remaining component pair NRTL parameters, the following adjustments were made to compensate for the small data set.

- Byproduct parameters were only regressed for component pairs involving the main solvent system, the product and the impurity. No Byproduct / Byproduct combinations of component pairs were regressed. As the majority of Byproducts move between the washes and back extraction together, this was expected to have minimal impact

- Byproduct 3 parameters were not regressed at all due to low quality of the data. The NRTL parameters available within Aspen for the components pairs involving the main solvent system were used instead, although it should be noted that these were based on VLE data. As this component was only present at low levels, this was expected to have minimal impact.

In addition, no dissociation or protonation was accounted for by assigning $\mathrm{pKa}$ values. This is a necessary simplification given the overall complexity of the system and large number of components involved, and is acceptable as the workup is carried out in neutral solvents. Whilst some of the compounds involved are weakly basic, no acidic species are present in the system and therefore there is minimal opportunity for protonation. It should also be remembered that the aim of the modeling work was not to accurately predict the behaviour of every single component; only to fit the partitioning of the product and impurity with sufficient accuracy to identify the optimal conditions and determine the impact on changing process parameters. The acceptable fit in the modeling work and its successful use to determine an optimized set of conditions demonstrates that this aim has been met and therefore that the simplification is appropriate.

If a process in future applied a wash containing an ionic solute, involved weak acids/bases, or relied on changes in protonation to move compounds between phases, this simplification may not be appropriate and rigorous modeling of dissociation and/or pKa using an alternative modeling approach could be required; this is out of scope of this publication. 
The resulting parameters are shown in Table $\mathbf{S 7}$ below.

Table S7. Regressed NRTL model parameters for the remaining components at ambient temperature. C parameter values were not regressed but were fixed at the value shown in the table.

\begin{tabular}{|l|l|l|l|l|}
\hline Component 1 & Component 2 & \multicolumn{1}{|c|}{$\mathbf{A}_{\mathbf{i j}}$} & \multicolumn{1}{|c|}{$\mathbf{A}_{\mathbf{j i}}$} & \multicolumn{1}{c|}{$\mathbf{C}$} \\
\hline Water & Byproduct 1 & -3.184 & -3.462 & 0.3 \\
\hline Toluene & Product & -0.970 & -0.945 & 0.3 \\
\hline Water & Product & 0.524 & 0.856 & 0.3 \\
\hline Toluene & Impurity & -5.030 & -3.223 & 0.3 \\
\hline Ethanol & Impurity & -21.574 & 41.179 & 0.3 \\
\hline Water & Impurity & 4.182 & -10.17 & 0.3 \\
\hline Ethanol & Product & 2.679 & 3.122 & 0.3 \\
\hline Byproduct 4 & Product & 3.461 & 0.798 & 0.3 \\
\hline Toluene & Byproduct 4 & 10.393 & 1.405 & 0.3 \\
\hline Water & Byproduct 4 & 6.960 & 3.393 & 0.3 \\
\hline Ethanol & Byproduct 4 & -1.975 & 10.966 & 0.3 \\
\hline Toluene & Byproduct 1 & -2.707 & -2.932 & 0.3 \\
\hline Ethanol & Byproduct 1 & 0.032 & 2.366 & 0.3 \\
\hline Byproduct 1 & Product & 5.100 & 1.276 & 0.3 \\
\hline Byproduct 2 & Product & 2.586 & 3.199 & 0.3 \\
\hline Byproduct 4 & Impurity & 16.877 & -3.856 & 0.3 \\
\hline Byproduct 1 & Impurity & 13.414 & -12.140 & 0.3 \\
\hline Byproduct 2 & Impurity & -20.587 & -7.401 & 0.3 \\
\hline
\end{tabular}

Overall, these parameters gave a good fit to the experimental data for all data points except three in which the level of toluene predicted in the aqueous phase was too high, with a subsequent impact on the level for all other components. The reason for this is not clear; however, the fit was deemed sufficient to proceed to verification. 


\section{MATLAB R2018b Image Analysis Code}

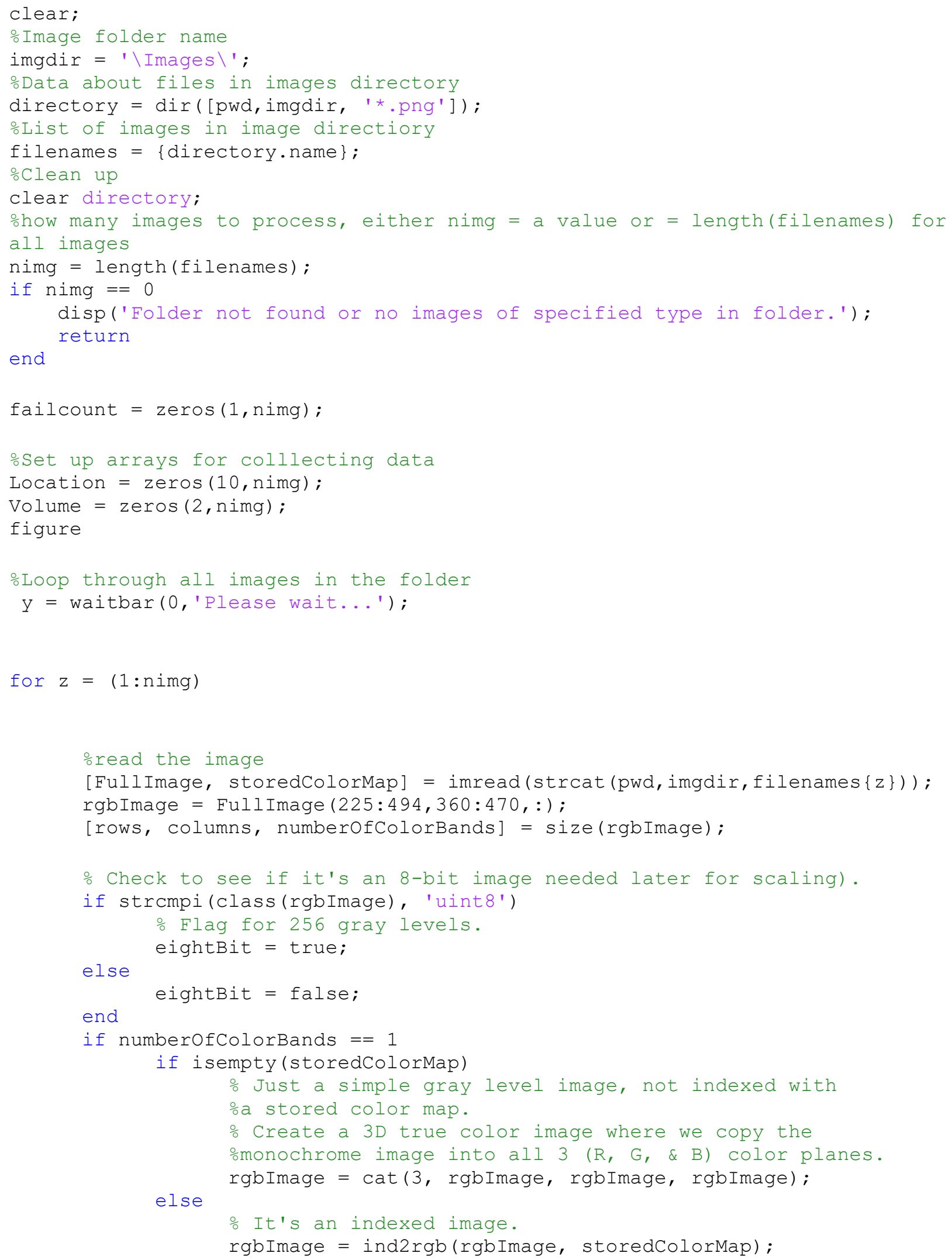




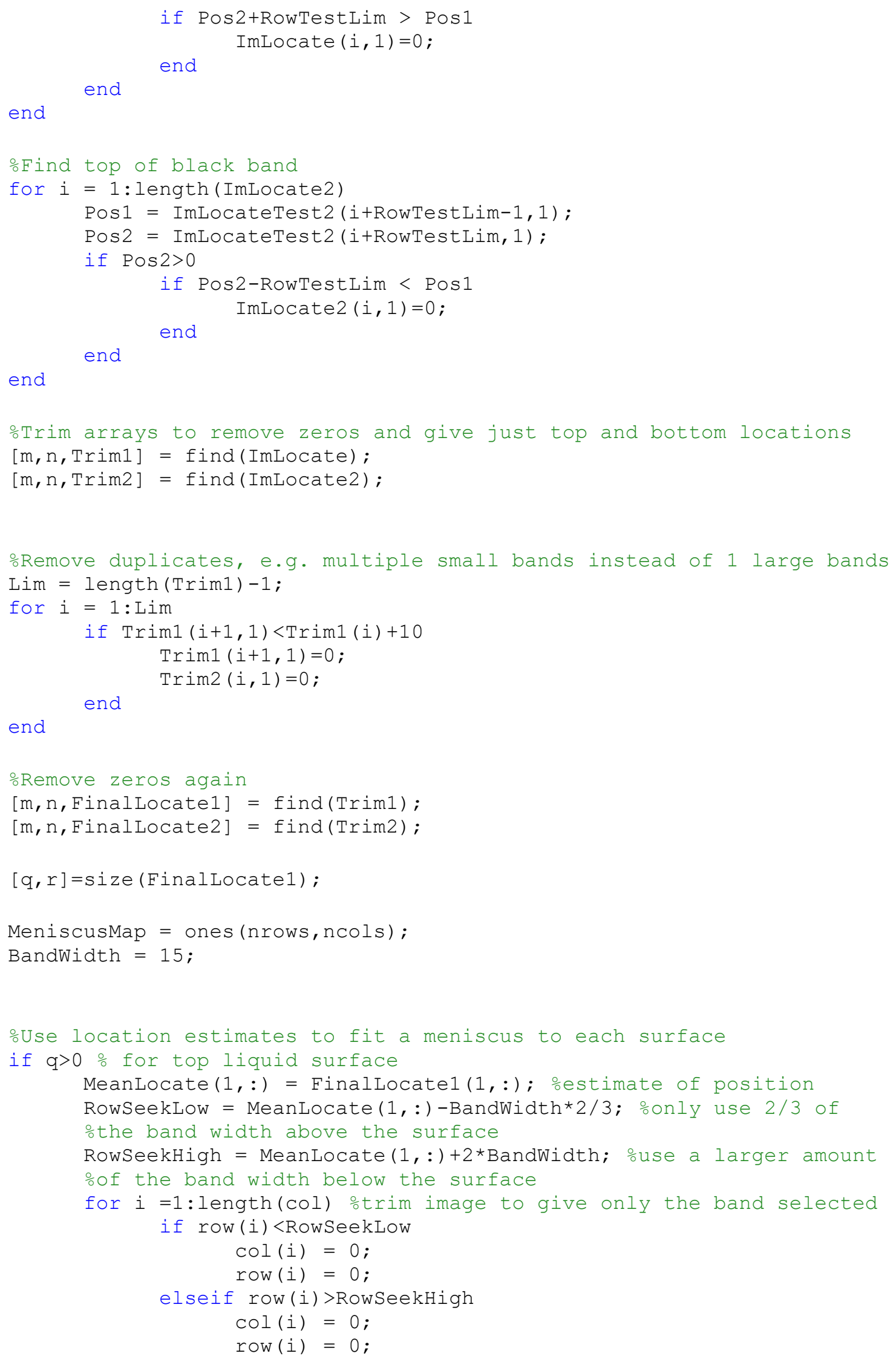




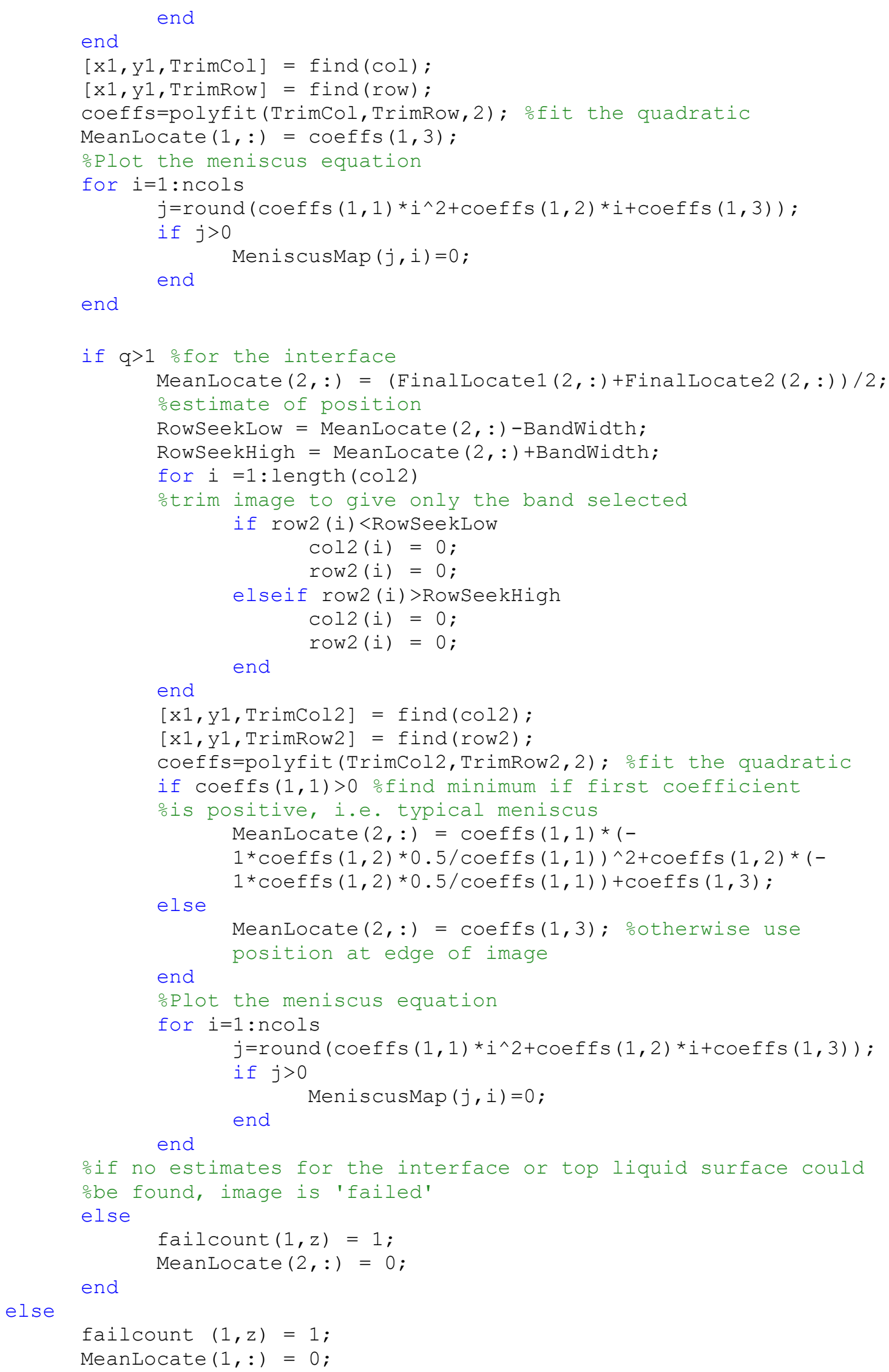




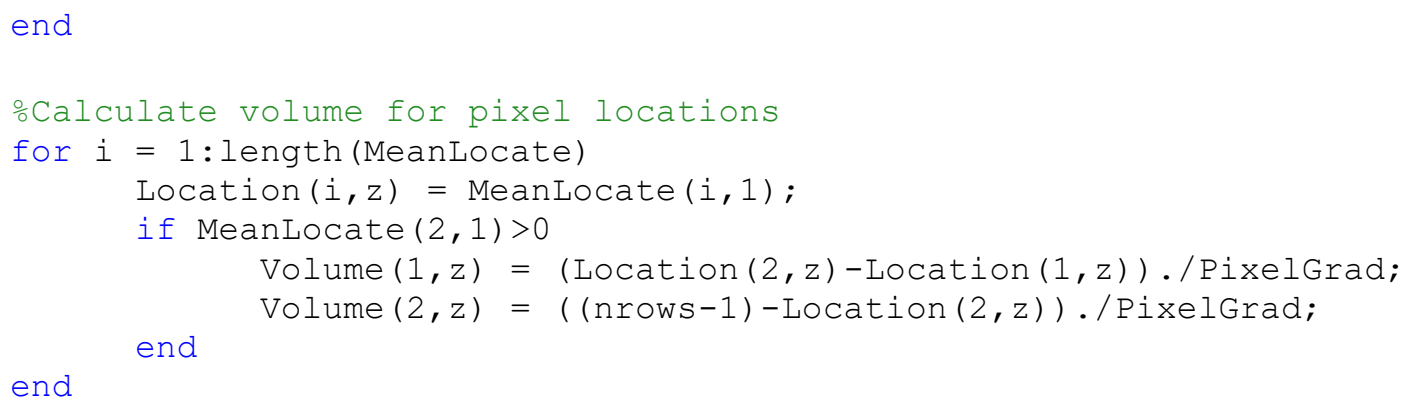

Volume 